Nobuyuki Shimozawa $\cdot$ Yasuyuki Suzuki $\cdot$ Zhongyi Zhang Kiyokuni Miura • Akiko Matsumoto • Masahiro Nagaya Silvia Castillo-Taucher $\cdot$ Naomi Kondo

\title{
A novel nonsense mutation of the PEX7 gene in a patient with rhizomelic chondrodysplasia punctata
}

Received: October 16, 1998 / Accepted November 11, 1998

\begin{abstract}
Mutations in the $P E X 7$ gene encoding a peroxisome targeting signal 2 (PTS2) were identified in two patients with rhizomelic chondrodysplasia punctata (RCDP). A 7-year-old girl, the first Japanese individual to be diagnosed biochemically as a case of RCDP, had a novel nonsense mutation, R232ter, in the PEX7 gene, which had been inherited from her consanguineous parents. Another patient, a Chilean boy with RCDP, had compound heterozygous mutations of PEX7, L292ter and A218V, both of which have been documented. R232ter, which deletes all of the last two WP40 repeats in the $P E X 7$ gene, is sufficient to inactivate functions of the $P E X 7$ gene.
\end{abstract}

Key words Rhizomelic chondrodysplasia punctata $P E X 7$. Peroxisome targeting signal 2

\section{Introduction}

Rhizomelic chondrodysplasia punctata (RCDP; McKusick 215100) is a fatal autosomal recessive disorder characterized by the presence of calcific stippling of multiple joints, disproportionately short stature with symmetrical shortening of proximal extremities, typical craniofacial dysmorphism, and severe mental and growth retardation. Biochemical abnormalities in RCDP include deficiency of acyl-coenzyme A (CoA), dihydroxyacetone phosphate acyl transferase (DHAPAT), alkyl-dihydroxyacetonephosphate synthase (alkyl-DHAP synthase), and phytanoyl-CoA hydroxylase, and the absence of the mature form of peroxiso-

N. Shimozawa $(\bowtie) \cdot$ Y. Suzuki $\cdot$ Z. Zhang $\cdot$ N. Kondo

Department of Pediatrics, Gifu University School of Medicine, 40

Tsukasa-cho, Gifu 500-8076, Japan

Tel. +81-58-265-1241; Fax +81-58-265-9011

e-mail: nshim@ cc.gifu-u.ac.jp

K. Miura $\cdot$ A. Matsumoto $\cdot$ M. Nagaya

Aichi Prefectural Colony Hospital, Kasugai, Japan

S. Castillo-Taucher

Servicio de Genetica, Hospital Clinico Universidad de Chile,

Santiago, Chile mal 3-ketoacyl-CoA thiolase (PT) (Lazarow and Moser 1995). The gene involved, human PEX7, which encodes the receptor of peroxisome targeting signal (PTS) 2, was identified, and its mutations, L292ter, G217R, and A218V were identified in RCDP patients (Braverman et al. 1997, Motley et al. 1997, Purdue et al. 1997). We now report a novel nonsense mutation in a 7-year-old girl, the only Japanese individual to have been diagnosed biochemically. We also report compound heterozygote mutations noted in a Chilean patient.

\section{Patients and methods}

Patient 1

This 7-year-old girl is the first-born child of Japanese parents who are second cousins, and has clinical and biochemical abnormalities characteristic of RCDP, including DHAPAT deficiency and the precursor form of PT (Suzuki et al. 1993). Psychomotor retardation in this girl is severe.

\section{Patient 2}

This 7-year-old Chilean boy has the typical RCDP phenotype and inversion of chromosome 8 (Castillo-Taucher et al. 1991). Biochemical analysis revealed a deficiency in DHAPAT activity (patient $2,0.59 \mathrm{nmol} / \mathrm{mg}$ per $2 \mathrm{~h}$; patient $1,0.35 \mathrm{nmol} / \mathrm{mg}$ per $2 \mathrm{~h}$; control, $1.99 \pm 0.18 \mathrm{nmol} / \mathrm{mg}$ per $2 \mathrm{~h}$ ), and the presence of the precursor form of PT on immunoblotting (data not shown). Psychomotor retardation is also severe in this boy.

To search for mutations in the $P E X 7$ gene, we made use of reverse transcription-polymerase chain reaction (RTPCR) to amplify PEX7 cDNA (GenBank accession numbers: U88871) from fibroblast RNA isolated from the two patients. We used primers 5'-GATGAGTGCGGTGTGCGGTG-3' (forward) and 5'-ACTCCTGCTGCTTCACATC-3' (reverse) to obtain a 570-bp product, primers 
5'-TTTTGCTTCAGCCTCAGGTG-3' (forward) and 5'ATAGGTATGACCAAGAAGTT-3' (reverse) to obtain a 220-bp product, and primers 5'-GGAATGTACGACAACCAGTG-3' (forward) and 5'-GACCAAAGTAGTGTATCTCA-3' (reverse) to obtain a 304-bp product. The amplified DNA fragments were directly sequenced using an automated DNA sequencer. Genomic DNA was obtained from the fibroblasts from patient 1 , and blood samples were taken from her parents, followed by PCR amplification, using primers 5'-GGGCGGTTGACTGTAGTTTG-3' (forward) and 5'-ATAGGTATGACCAAGAAGTT-3' (reverse) to obtain a 80-bp product containing the R232 codon. DHAPAT activities and immunoblotting were performed as described (Shimozawa et al. 1988a, Shimozawa et al 1988b).

\section{Results and discussion}

Direct sequencing of PCR amplified PEX7 cDNA from patient 1 revealed a C-to-T transition at position 694 (hereafter, starting from the first nucleotide of the initiator methionine codon), which led to an Arg232-to-termination codon (Fig. 1). Only this mutation was observed at the position of cDNA, so we next determined the mutation in genomic DNA from the patient and her consanguineous parents. The parents were shown to be heterozygous for the mutation (Fig. 1), and the patient was homozygous for this mutation (data not shown). This suggests that patient 1 inherited the R232ter mutation from her parents.

The PEX7 cDNA of patient 2 contained compound heterozygous mutations of C-to-T transition at position 635, resulting in Ala218-to-Val and a T-to-A transition at position 875, resulting in Leu292-to-termination codon (data not shown); both of these mutations have been reported (Braverman et al. 1997, Motley et al. 1997, Purdue et al. 1997).

PEX7 is the PTS2 receptor containing six consecutive WD40 motifs, and rescues PTS2 import in fibroblasts from RCDP patients (Braverman et al. 1997, Motley et al. 1997, Purdue et al. 1997). The L292ter mutation is frequent among RCDP patients and severely impairs functions of the PEX7 gene (Brites et al. 1998). This mutation deletes nearly all of the last WD40 repeat and the additional downstream sequence in the $P E X 7$, which could inactivate $P E X 7$ function. The newly identified R232ter mutation truncates 60 amino acids shorter than the R292ter, and deletes all of the last two WD40 repeats. This suggests that, in patient 1 , the only Japanese patient to be diagnosed biochemically as a case of RCDP, the condition is caused by the homozygous R232ter mutation in $P E X 7$, inherited from her parents.

Acknowledgements We thank K. Hori for technical assistance and M. Ohara for helpful comments. This study was supported in part by a Grant-in-Aid for Scientific Research (10670721) from the Ministry of Education, Science, Sports, and Culture of Japan, by a research grant from the National Center of Neurology and Psychiatry of the Ministry of Health and Welfare of Japan, and by a research grant from the ONO Medical Research Foundation.

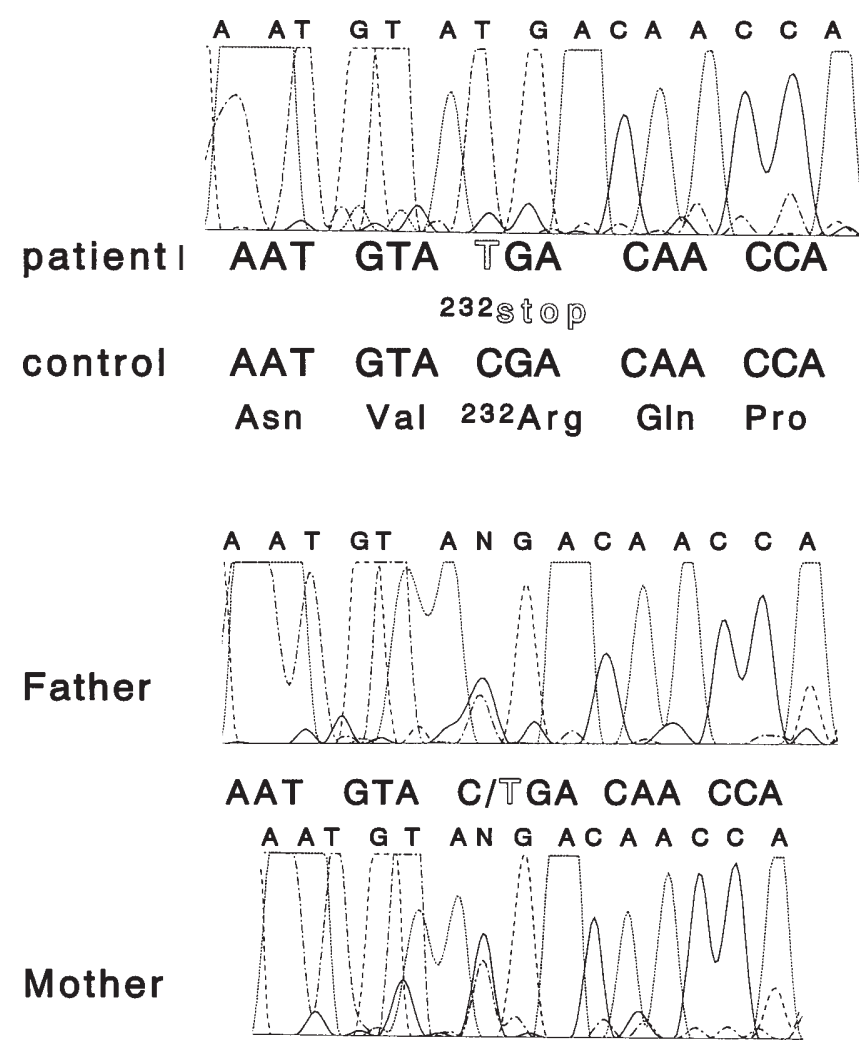

Fig. 1 Sequence analysis of PEX7 cDNA and genomic DNA containing the R232ter mutation from patient 1 and her parents, respectively. The mutation of the C-to-T transition, resulting in the Arg232-totermination codon, was detected in patient 1 , homozygously, and in the parents, heterozygously

\section{References}

Braverman N, Steei G, Obie C, Moser A, Moser H, Gould SJ, Valle D (1997) Human PEX7 encodes the peroxisomal PTS2 receptor and is responsible for rhizomelic chondrodysplasia punctata. Nat Genet 15 : 369-376

Brites P, Motley A, Hogenhout E, Hettema E, Wijburg F, Heijmans HSA, Tabak HF, Distel B, Wanders RJA (1998) Molecular basis of rhizomelic chondrodysplasia punctata type I: High frequency of the Leu-292 stop mutation in 38 patients. J Inherit Metab Dis 21: 306308

Castillo-Taucher S, Baca JP, Saez R, Geldres V (1991) Balanced pericentric inversion 8 (p23q13) in a child with rhizomelic chondrodysplasia punctata and his mother. Clin Genet 40: 247248

Lazarow PB, Moser HW (1995) Disorders of peroxisome biogenesis. In: Scriver CR, Beaudet AL, Sly WS, Valle D (eds) The metabolic and molecular basis of inherited disease. 7th edn. McGraw-Hill, New York, pp 2287-2324

Motley AM, Hettema EH, Hogenhout EM, Brites P, ten Asbroek ALMA, Wijburg FA, Baas F, Heijmans HS, Tabak HF, Wanders RJA, Distel B (1997) Rhizomelic chondrodysplasia punctata is a peroxisomal protein targeting disease caused by a non-functional PTS2 receptor. Nat Genet 15: $377-380$

Purdue PE, Zhang JW, Skoneczny M, Lazarow PB (1997) Rhizomelic chondrodysplasia punctata is caused by deficiency of human PEX7, a homologue of the yeast PTS2 receptor. Nat Genet 15: 381384

Shimozawa N, Suzuki Y, Orii T, Yokota S, Hashimoto T (1988a) Biochemical and morphologic aspects of peroxisomes in the human rectal mucosa: Diagnosis of Zellweger syndrome simplified by rectal biopsy. Pediatr Res 24: 723-727 
Shimozawa N, Suzuki Y, Orii T, Hashimoto T (1988b) Immunoblot detection of enzyme proteins of peroxisomal beta-oxidation in fibroblasts, amniocytes, and chorionic villous cells. Possible marker for prenatal diagnosis of Zellweger's syndrome. Prenat Diagn 8: 287-290
Suzuki Y, Shimozawa N, Izai K, Uchida Y, Miura K, Akatsuka H, Nagaya M, Yamaguchi S, Orii T (1993) Peroxisomal 3-ketoacyl-CoA thiolase is partially processed in fibroblasts from patients with rhizomelic chondrodysplasia punctata. J Inherit Metab Dis 16: 868-871 